\title{
Analysis on the Spatial Pattern Evolution of Chinese Provincial Environment-Energy Efficiency Based on ESDA-GIS ${ }^{1}$
}

\author{
Xiaoting LIU ${ }^{1}$, Wenjun CHEN ${ }^{2}$ \\ ( Institute of Economics, Xinjiang University of Finance and Economics, Urumqi 830012, China)
}

\begin{abstract}
Keywords: China; Environment - energy efficiency; Spatial Pattern; ESDA-GIS; The standard deviational ellipses
\end{abstract}

Abstract: By virtue of window analysis and by using DEAP2.1 software, this paper first calculates China's provincial domain environment- energy efficiency.Then, by using the spatial statistical software ArcGIS9.3 and exerting the combined method of ESDA and Standard Deviational Ellipses, it analyzes Chinese provincial environment - energy efficiency's spatial pattern evolution characteristics to reveal that China's environment - energy efficiency has a significant spatial auto-correlation and that the area coverage of China's environment - energy efficiency's hot and sub hot spots has been expanding gradually while that of cold and sub cold spots has been shrinking unceasingly since 2001. During the period of 2000-2012, the standard deviational ellipses of Chinese provincial environment - energy efficiency presents the northeast - southwest spatial distribution pattern as it gradually shifts towards east - west direction. On the whole, the main part of environment-energy efficiency changes from northeast, northwest to southwest direction, with the junction of Henan and Hubei province as the center and covering provinces that are relatively advanced in economy and technology. The dynamic evolution characteristics of Chinese provincial environment - energy efficiency spatial pattern enlightens the government to take a green, low carbon, cyclic development road under the "new normal" background, to pay more attention to the function of spatial correlation.

During the period of October 2014 to March 2015, some cities in China appeared serious smog weather, the broad coverage and serious degree of which caused the whole nation to pay high attention to the economic development and environmental protection. Superficially, smog was considered a product of adverse weather conditions, but the root cause has been determined to be due to long-term one-sided pursuit of GDP, ignoring the environment and unreasonable structure of energy accumulation, etc. Problems like imbalanced economic development, inconsistent energy efficiency level and serious environmental pollution impinge on the survival of mankind. At present, many domestic scholars focus on China's energy efficiency measure, evaluation and influence factors' research[1-3]. There are many scholars focusing on analyzing regional differences in China's energy efficiency and the potential analysis of energy conservation and emissions reduction[4-5]. And many other scholars, after drawing lessons from foreign scholars about spatial econometric methods and models, also studied on spacial differences of energy efficiency. Among those researches, there are representative researches, such as Yanfen Zou, Yuhai Liu [6] and Zhenyu Gao [7]. Using a spatial auto correlation model, The former two scholars analyzed the regional difference characteristics of China's energy efficiency and found the significant spatial auto correlation and heterogeneity between region's energy efficiency and economic development while the latter two, by using space planning method, divided China region's energy efficiency during the period of 1995-2003 into three areas: energy efficiency, energy middle efficiency, energy inefficiency through clustering analysis.

Through review of the existing literature, it is not difficult to find that research on energy and environmental problems exhibits certain limitation. In that much of it focus on only one aspect. They may only study area's energy efficiency, or focus on the area's environmental efficiency, and they just bring environmental factors into the calculation of the energy efficiency as part of it. However, research only considering energy consumption or environmental pollution is one-sided, and can not 
fully reflect the efficiency of regional economic development. Therefore, the environment-energy efficiency which comprehensively considering the energy consumption and environmental pollution is a more comprehensive and more effective evaluation index of regional efficiency. While research on environment-energy efficiency conducted thorough analysis on the time series data, it overlooked the analysis on the cross section data having spatial dependence effects. Therefore, taking the inter-dependencies relation between individuals in space into consideration, scientifically comparing Chinese provinces' environment-energy efficiency level, and analyzing the spatial distribution correlation, cold-hot spot evolution pattern and the spatial difference evolution characteristics of China's environment-energy efficiency will have important practical significance and the value of policy guidance.

\section{The Study Area, Data sources and Environment-Energy Efficiency Measurement}

\section{Survey Region and Data sources}

Due to the lack of the individual years data of Hong Kong special administrative region, Macao special administrative region and Taiwan province, Tibet autonomous region, the scope of this research includes only 30 provinces (as shown in figure 1). This article selects the 2000-2012 environment - energy efficiency as a research index, using data derived from the China Statistical yearbook (2002-2014) and the China energy statistical yearbook (2002-2014).

\section{Environment-Energy Efficiency Measurement}

This study, based on the $\mathrm{BC}^{2}$ model which features investment-oriented scale and constant return, with capital, labor, energy consumption as inputs, with environmental pollution (waste water, waste gas, solid waste) as the unfavorable output items, and GDP as desirable output, measures and calculates Chinese provinces domain environment - energy efficiency of 2000, 2004, 2008, 2012 with the aid of window analysis and the use of DEAP2.1 software.

\section{Research methods}

\section{The overall evolution characteristics analysis method}

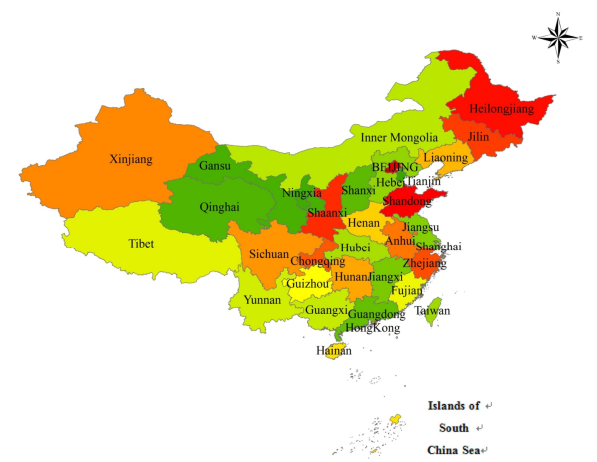

Fig. 1 Research Region

Global Moran' s I index reflects the similarity of unit properties about space adjacent areas, the formula (1) is showed as follows:

$$
I=\frac{\sum_{i=1}^{n} \sum_{j=1}^{n} w_{i j}\left(x_{i}-\bar{x}\right)\left(x_{j}-\bar{x}\right)}{S^{2} \sum_{i=1}^{n} \sum_{j=1}^{n} w_{i j}} \quad \text { (1) } \quad Z(I)=\frac{I-E(I)}{\sqrt{\operatorname{var}(I)}}
$$

Letter I represents for Moran index, $\mathrm{n}$ for the number of sample areas, $\mathrm{x}_{\mathrm{i}}$ for the attribute values of region $\mathrm{i}, \mathrm{x}_{\mathrm{j}}$ for the attribute values of adjacent region $\mathrm{j}$ to region $\mathrm{i} . \bar{x}$ for the average of all property values. $\mathrm{W}_{\mathrm{ij}}$ is used to measure the spatial autocorrelation weights, which show the relationship between $\mathrm{i}$ and $\mathrm{j}$. If region $\mathrm{i}$ and $\mathrm{j}$ are adjacent to each other, then $\mathrm{w}_{\mathrm{ij}}=$ one, otherwise, $\mathrm{w}_{\mathrm{ij}}=$ zero. The value of Global Moran's I index is between $[-1,1]$. If it is greater than zero, it indicates positive correlation; Otherwise, for negative correlation; if it equals to zero or near zero, it means that the space areas are not related. This article use the formula (2) to do statistical test on the Global Moran' s I results. Among these, var (I) is the variance of I. When the statistics Z of Moran' s I is greater than the critical value, that is, 1.96 , of normal distribution function at the 0.05 level, it will be considered to be significant [9]. 


\section{Local Evolution Characteristics Analysis Method}

Getis- Ord Gi* is used to identify the spatial distribution of cold and hot area. The design formula is shown as below [10]:

$$
G_{i}^{*}(d)=\sum_{j=1}^{n} W_{i j}(d) X_{j} / \sum_{j=1}^{n} X_{j} \quad \text { (3) } \quad Z\left(G_{i}^{*}\right)=\frac{G_{i}^{*}-E\left(G_{i}^{*}\right)}{\sqrt{\operatorname{Var}\left(G_{i}^{*}\right)}}
$$

For convenience of comparison and analysis, this paper has done standardizing processing according to formula (3) and the processing result is as formula (4). Among them, $\mathrm{E}\left(\mathrm{Gi}^{*}\right)$ is as the mathematical expectation, $\operatorname{Var}\left(\mathrm{Gi}^{*}\right)$ is as the coefficient of variation, $\mathrm{W}_{\mathrm{ij}}(\mathrm{d})$ as the spatial weight. If $\mathrm{Z}$ $\left(\mathrm{Gi}^{*}\right)$ is positive and significant, then it suggests that position $\mathrm{i}$ has a high value, and the adjacent value is higher, which belongs to the hot zone; On the other hand, if the $\mathrm{Z}$ ( $\mathrm{Gi} *$ ) is negative and significant, it explains that the value of the position $i$ has a lower value and the adjacent value is also lower, which belongs to the cold spot area.

The Standard Deviational Ellipses ( abbreviated as SDE) is one of the important spatial econometric analysis methods which is used to measure the spatial differences between regional economic elements and analyze spatial distribution characteristics of economic elements[11]. The main elements include: center of gravity (center point), corner $\theta$, semi-major axis, semi-minor axis. Among them, the center of gravity represents the relative position of the spatial distribution of regional economic elements, demonstrated by the calculation formula below:

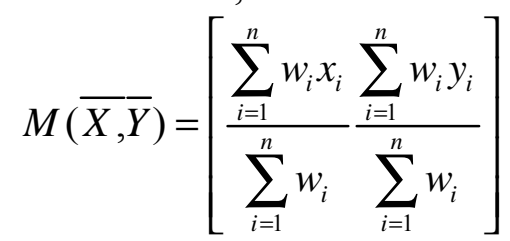

$(\bar{X}, \bar{Y})$ is the regional center of gravity, $\mathrm{n}$ is the number of region, $w_{i}$ is the weight of the $\mathrm{i}^{\text {th }}$ subset area and some attribute values, (xi, yi) is the center coordinates of the $i^{\text {th }}$ subset area.Corner $\theta$ is the included angle formed by due north direction clockwise turning to the long axis of the ellipse, which indicates the main trend of the spatial distribution of economic elements with calculation formula as below:

$$
\begin{array}{ccc}
\tan \theta=\frac{A+B}{C} & \text { (6) } & \mathrm{A}=\sum_{i=1}^{n} \tilde{x_{i}^{2}}-\sum_{i=1}^{n} \tilde{y_{i}^{2}} \\
B=\sqrt{\left(\sum_{i=1}^{n} \tilde{x_{i}^{2}}-\sum_{i=1}^{n} \tilde{y_{i}^{2}}\right)^{2}+4\left(\sum_{i=1}^{n} \tilde{x_{i}} \tilde{y_{i}}\right)^{2}} & \text { (8) } & C=2 \sum_{i=1}^{n} \tilde{x_{i}} \tilde{y}_{i},
\end{array}
$$

$\tilde{x}_{i}$ and $\tilde{y_{i}}$ is the deviation between coordinates of the $i^{\text {th }}$ subset area and center of gravity. The semi-major axis reflects the discrete degree of economic factors on the main trend direction while the semi-minor axis reflects the discrete degree of economic factors on the minor trend direction.

\section{Empirical Analysis on Dynamic Evolution of Environment - Energy Efficiency Spatial Pattern \\ Overall Dynamic Evolution}

Based on the formula (1), this paper calculates the Global Moran's I value of China's 30 provinces' environment-energy efficiency by using the ArcGIS9.3 software as well as the corresponding value of $Z$ and $P$ (as shown in table 1 ).

From table 1, it is clear that during the period of 2000-2012, the value of Z - Scores are all greater than 1.96, and the corresponding value of Moran's I are also relatively large, which indicates that in 2000-2012, China's provincial environment -

Table1 Moran' s I statistics of China's provincial environment - energy efficiency

\begin{tabular}{lcccc}
\hline Statistical value & 2000 & 2004 & 2008 & 2012 \\
\hline Moran' s I & 0.2792 & 0.4018 & 0.4187 & 0.3445 \\
Z-Score & 2.0845 & 2.8996 & 3.4103 & 2.4296 \\
P-value & 0.0482 & 0.0358 & 0.0321 & 0.0387 \\
\hline
\end{tabular}
energy efficiency level has a significant positive spatial correlation. According to the data of P - value, it shows that the $\mathrm{P}$ value of each year is less than 0.05 , explaining that it has passed significant test 
during the study period. The value of Moran's I shows a rapid decline in 2012, at the same time the significative degree of positive correlation is decreased, which show that spatial difference of different provincial environment -energy efficiency is increasing gradually.

From the figure 2, the tests of Moran's I and Z Score are almost consistent with each other as time changes, both presenting an inverted "V" shape, that is, during 2000-2008, there is a process of gradual rise, and the period of 2008-2012 shows a trend of gradual decline. Global Moran's I Index only reveals the global comprehensive characteristics of Chinese provincial environment energy efficiency, but it is still not enough to explain that each province has the same situation. That's why we need to conduct deep analysis on China's provincial environment - energy efficiency by combining Getis - Ord Gi * index and standard

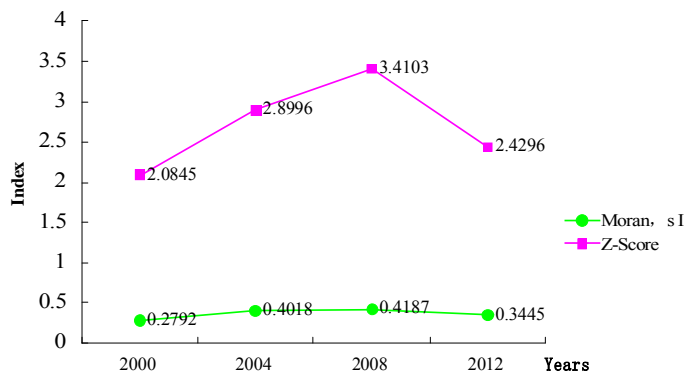

Fig.2 2000-2012 test changing chart of China's provincial environment-energy efficiency's Moran's I and Z-Score deviational ellipses.

\section{Local Dynamic Evolution}

The Getis - Ord Gi * Index Analysis. According to formula (3) and (4), the ArcGIS9.3 software is used to calculate the value of Getis - $\mathrm{Ord} \mathrm{G}_{\mathrm{i}}{ }^{*}$ index of 2000 and 2012. Then the optimum natural fracture method of Jenks is used to divide the local Getis - word $\mathrm{Gi}$ * index value of the two year into four classes: hot spot region, sub-regional hot zone, sub-regional cold zone and cold spot region by descending order. Finally the hot spot evolving graph of spatial pattern of China's environment energy efficiency is generated(as showed by Figure 3)

In this case, figure 3 shows twosignificant points. (1) In 2000, hot spot and sub-hot zone of China's provincial environmental-energy efficiency are mainly distributed in north China, east China, southwest China, while cold zone are mainly distributed in Xinjiang, Heilongjiang, Jilin province. In 2012, hot zones of China's provincial environmental-energy efficiency further expand to Guangxi and Guangdong Provinces, while cold spot and sub-regional cold zone present shrinking trend which indicates that China's provinces environment - energy efficiency has been improved. But on the whole, China's environment - energy efficiency presents a spacial change pattern that is "South higher than North" and "East higher than West". The difference of each province's environment-energy efficiency is significant and China still has a long way to go for development that is green, low carbon, cyclic in the future.(2) During the period of 2000-2012, provinces whose environment-energy efficiency has been in a cluster of high value are Shanxi, Henan and Hubei and provinces that are in a cluster of low value are Heilongjiang, Jilin, Xinjiang. From the perspective of the evolution of hot zone and cold zone, hot zoen of environment-energy efficiency mainly concentrates in the central region in 2001, and as hot zone scope expands gradually, Shanxi province in northwestern region fall into hot zone. During the research period, there is no significant change about the cold zone. This characteristics of spatial pattern evolution of environment-energy efficiency is mainly and comprehensively decided by economic development level, technology level, energy consumption structure, industrial structure and population size of each province.

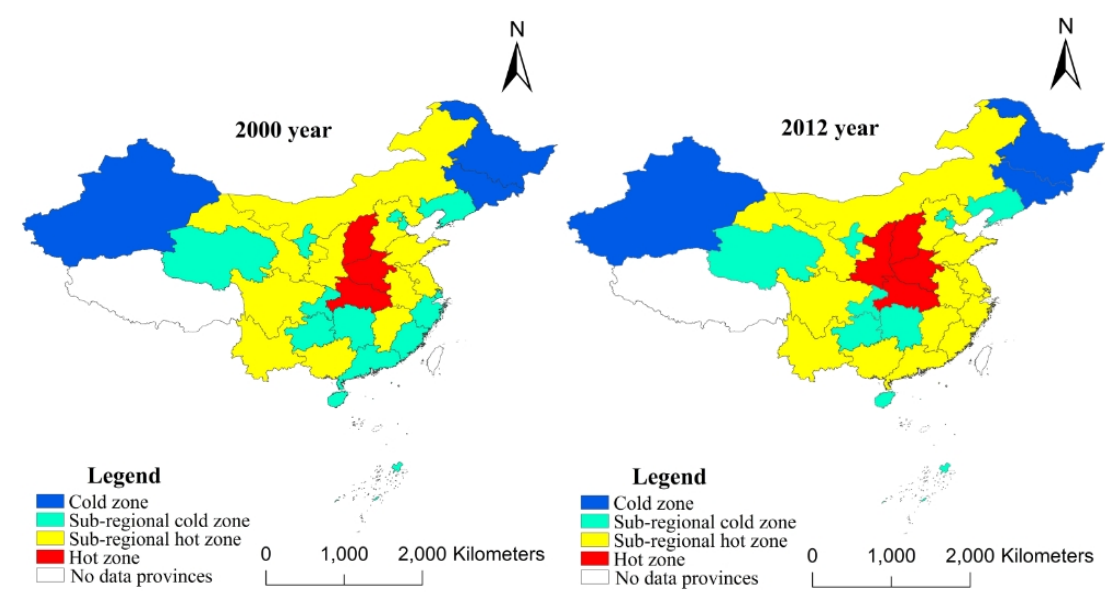

Fig.3 The evolution Chart of China's Provincial Environment-Energy Efficiency Hot Zone (2001 and 2012) 


\section{Standard Deviational Ellipses Analysis}

The calculation for the standard deviational ellipses parameter of China's provincial environment energy efficiency in 2000-2012 are shown in table 2, and the spatial distribution situation is shown in figure 4.

In figure 4, it is clear that the center of China's provincial environment - energy efficiency during the research period is always at the junction of Henan province and Hubei province. In 2000-2012, the standard deviation ellipse has a larger amplitude of fluctuation and the coverage range of it is east to Shandong, Jiangsu, Shanghai, Zhejiang, Fujian, west to Sichuan, Qinghai, Gansu, north to Inner Mongolia and south to Guangxi, Guangdong, which basically covers China's provinces having relatively developed economies. In 2000-2012, the standard deviation ellipse distribution range shows that the environment - energy efficiency appears as space expansion trends. Combined with the evolution figure of provincial environmental-energy efficiency hot zone, we can find that hot zone and sub-regional hot zone are basically located within the standard deviation ellipse while the cold zone and sub-regional cold zone are all located outside the standard deviational ellipses.

From the variation range of the intersection angle $\theta$, during the period of 2000-2012, the intersection angle basically between basic change within the scope of $60.068^{\circ}-67.829^{\circ}$. On the whole, the spatial pattern distribution of China's provincial environment-energy efficiency presents a pattern that is from northeast to southwest. Among them, the intersection angle $\theta$ narrows from $66.884^{\circ}$ to $60.702^{\circ}$ during $2000-2004$, which weakens the northeast - southwest spatial pattern. While during 2008-2012, the rotation angle expands from $60.068^{\circ}$ to $67.829^{\circ}$, which strengthen the northeast - southwest pattern.

Seen from the principal axis direction, in 2000-2008, the standard deviation of the main semiaxis expands from $11.886 \mathrm{~km}$ to $12.369 \mathrm{~km}$, indicating that the provincial environment-energy efficiency during that period disperses in a northeast - southwest direction ; In 2008-2012, the standard deviation of the main semiaxis declines from $12.369 \mathrm{~km}$ to $12.062 \mathrm{~km}$, showing that China's environment - energy efficiency appears a spatial polarization in the northeast - southwest direction. From the point of view of an auxiliary axis direction, the standard deviation of the auxiliary semiaxis goes down from $9.366 \mathrm{~km}$ in 2000 to $9.352 \mathrm{~km}$ in 2004 , indicating that during that study period, provincial

Table2 Standard deviational ellipses of China's provincial Environment-energy efficiency during 2000-2012

\begin{tabular}{ccccc}
\hline Years & 2000 & 2004 & 2008 & \multicolumn{1}{c}{2012} \\
\hline corner $\theta /{ }^{\circ}$ & 66.884 & 60.705 & 60.068 & 67.829 \\
Standard deviation along Y axis/km & 11.886 & 12.242 & 12.369 & 12.062 \\
Standard deviation along X axis $/ \mathrm{km}$ & 9.366 & 9.352 & 9.361 & 9.546 \\
\hline
\end{tabular}

environment - energy efficiency presents a spatial polarization phenomenon in the northwest southeast direction; The standard deviation of the auxiliary semiaxis goes from $9.361 \mathrm{~km}$ in 2008 up to $9.546 \mathrm{~km}$ in 2012 , which indicates that the provincial environment - energy efficiency during that period presents a spatial decentralization phenomenon in the northwest - southeast direction.

The results in calculation of the barycentric coordinates of China's environment - energy efficiency in 2000-2010 is as shown in table 3 . From the perspective of the spatial distribution of the center of gravity, the center of gravity during study period floats within the range of $112.918^{\circ}-113.258^{\circ} \mathrm{E}$, $32.331^{\circ}-32.569^{\circ} \mathrm{N}$, generally located at junction of Henan province and Hubei province. From the point of the center of gravity's moving trajectory, on the whole, the center of gravity shows a trend of

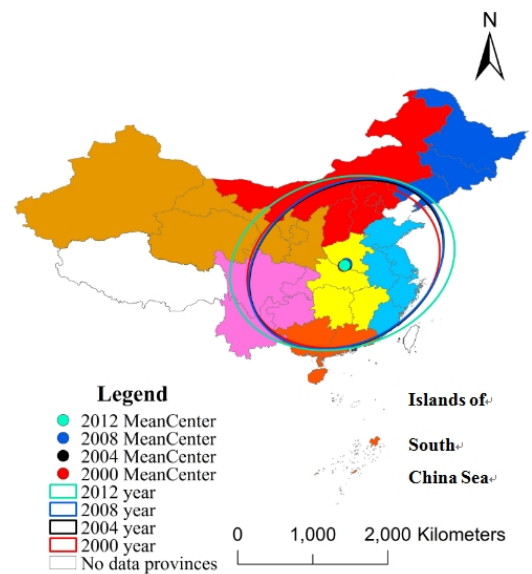

Fig.4 Distribution of mean center and standard deviational ellipses of China's environment-energy efficiency during 2000-2012 
changes that starts from northeast, to northwest then to southwest. More specifically, it moves 34.768 $\mathrm{km}$ to North-East during 2001-2004, and $27.032 \mathrm{~km}$ to the southwest during 2008-2012 showing that distance in the east-west direction is greater than in north-south direction.

Table 3 Shift of the center of gravity

of China's provincial environment-energy efficiency during 2000-2012

\begin{tabular}{lcccc}
\hline \multicolumn{1}{c}{ Years } & 2000 & 2004 & 2008 & 2012 \\
\hline Central coordinates & $112.918^{\circ} \mathrm{E}, 32.331^{\circ} \mathrm{N}$ & $113.258^{\circ} \mathrm{E}, 32.454^{\circ} \mathrm{N}$ & $113.256^{\circ} \mathrm{E}, 32.569^{\circ} \mathrm{N}$ & $113.047^{\circ} \mathrm{E}, 32.402^{\circ} \mathrm{N}$ \\
Moving direction & Northeast & Northwest & Southwest & - \\
Moving distance $/ \mathrm{km}$ & 34.768 & 12.803 & 27.032 & - \\
\hline
\end{tabular}

\section{Conclusion and discussion}

In 2000-2012 period, this study selected the Chinese provincial environment and energy efficiency data as a measure, using Global Moran' s I, Getis - word Gi * studied the space correlation between Chinese provincial environment and the energy efficiency, Adopted standard deviation ellipse reveals the space dynamic evolution characteristics of environment - energy efficiency, found that China's provincial environment - the energy efficiency of spatial pattern evolution presents a certain regularity since 2000 .

China's overall spatial pattern of environment-energy efficiency represents strong space correlation, with similar areas distributed collectively. Showing that some province with high(low) environment-energy efficiency are close to surrounding high(low) environment-energy province and this kind of spatial correlation showed a trend of increasing in time, at the same time, It appears that the energy efficiency of a provincial environment not only affected by their own characteristics, but also affected by its neighborhood. Thus,in all interprovincial regions, strengthening technical exchanges and cooperation across regions is of paramount importance. The developed regions should make full use of their demonstration and leading role, to speed up the transfer of energy conservation and emissions reduction technology to the backward area, assisting them in technological innovation. At the same time, the underdeveloped regions should also actively learn from the developed areas of energy conservation and emissions reduction quality, to develop a suitable development stragety for their own low-carbon cycle development programs. On the other hand, the government should not impose one size fits all solutions in the provinces' domain classification task of energy saving and emission reduction; Grasping the dynamic evolution characteristics of spatial pattern in environment - energy efficiency, by optimizing the industrial structure, to improve energy consumption structure, strengthen technological innovation in the field of energy saving and emission reduction, reduce government intervention, effectively play a role of market mechanism of resource allocation, redouble our efforts to the construction of ecological civilization, improve environment- energy efficiency for the clear water, blue sky, all making for economic growth based on steady improvement in environmental quality, efficient use in energy, improving economy in quality.

From the hot spot area of provincial environment - energy efficiency, since 2001, hot and second hot spots areas are expanding gradually while cold spots and second cold spots areas are shrinking. This phenomenon indicates that Chinese provinces environment - energy efficiency is improving. But as a whole, the gap between provinces in environment -energy efficiency space is significant, There's still a long way to go ahead in energy conservation and emissions reduction field of work. Eastern coastal developed areas of high environment- energy efficiency provinces in China should be taking high-new technology as the traction, speeding up to set up a two-way system of regular exchange and visit with those provinces of underdeveloped economy, low level environment energy efficiency, making advanced technology and management experience in energy saving and emission reduction in eastern coastal areas spread to the low efficiency area. At the same time, these low environment energy efficiency provinces must accelerate the pace of the optimization and upgrading in industrial structure, optimize the industrial structure, vigorously improve the proportion of the tertiary industry, and actively develop new and high technology industries and services, In the process of undertaking industrial transfer of technology from energy efficient provinces, We should adhere to stated goals of 
ecological environmental protection, for the high energy consumption, high pollution, high emissions of project must be reasonable setted up barriers to entry, prevent spread of pollution, and finally realize the green development of economy, low carbon development, cycles development.

From the analysis of standard deviational ellipses, 2000-2012, China's environmental energy efficiency focus at $112.918^{\circ}-113.258^{\circ} \mathrm{E}$, floating between $32.331^{\circ}-32.569^{\circ} \mathrm{N}$, At the junction of henan province and hubei province, It showed a trend of changes in the northeast- northwestsouthwest direction. In the period of study, the environment- energy efficiency standard deviation ellipse present a spatial pattern of northeast to southwest, and gradually spread to all around, Including the scope of China's developed province with high economic and technological level. The spindle direction (northeast - southwest) presents a polarization phenomenon, The auxiliary axis (northwest - southeast) presents dispersion phenomenon. The government should develop a scientific and reasonable economic energy policy with innovative ideas, to promote a coordinated development between China's economy, society and energy- environment.(1) Definite the direction and mission of coordinated development between energy, economy and environment, strengthen the "energy economy - environment" chain policy item by item with reasonable cohesion, in accordance with the "conservation, clean, green and efficient" principle, improve the scientific and reasonable energy utilization efficiency as a strategic orientation, to connect with the policy of sustainable economy development, reducing fossil energy consumption, and endourage construction of a clear water, blue sky, ecological civilization, to promote the coordinate and sustainable development of economy, energy, environment. (2) To strengthen the government funding for ecological civilization construction, increase the policy support of recycling economy development, clean production promotion, energy conservation and emissions reduction. Expand the subsidies policy of green products consumption .Enforce a performance evaluation system of environmental protection, to meet the requirement of an ecological civilization predicated on the index system, evaluation methods, rewards and punishment mechanism.

\section{Acknowledgements}

This work was financially supported by the National Social Sciences fund research projects (14BJL050).

\section{References:}

[1] S. X. Li, J. H. Cheng. Study on the Energy Efficiency of China and its Determinants. Statistical Research, 2008, 25 (10): 19-25.

[2] Z. Zhou. Analysis on the China's Energy Efficiency Measures and its Influence Factors. Statistical Observation, 2014 (3):84-86.

[3] M. Yang, F. X. Yang, X. P. Chen. On influencing factors affecting China's Energy Efficiency: An Empirical Test Based on the VEC Model. Resources Science, 2011,33 (1): 163-168.

[4] K. L. Wang, B. C. Yang, L. Yang. Analysis on China's Energy Efficiency and Potentials of Energy Conservation and Emissions Reduction from the Perspective of Environmental impact. Management Review, 2012, 24 (8):40-50.

[5] D. Shi. Regional Differences in China's Energy Efficiency and Conservation Potentials . China Industrial Economy, 2006 (10):49-58.

[6] Y. F. Zou, Y. H. Lu. Study on the regional characteristic of energy efficiency in China Based on the Spatial Sub Regression Model. Statistical Research, 2005,10(10):67-71.

[7] Z. Y. Gao, Y. Wang. Analysis on the Classification of China's Provinces according to Energy Productivity and its influencing factors. Quantitative Economic Technology Research, 2006 (9):46-57.

[8] C. X. Han, Y. F. Ma, H. Luo. Economic spatial evolution and its mechanism in Xinjiang . Arid Land Geography, 2010,33(3):449-455.

[9] Y. Shi, R. H. Mi. Differentiation of population spatial distribution in Shaanxi Province. Arid Land Geography, 2015,38 (2):368-376. 